\title{
Educação farmacêutica: a percepção de estudantes de especialização em farmácia clínica
}

Pharmaceutical education: the perception of post-graduation students in clinical pharmacy

Educación farmacéutica: la percepción de estudiantes de especialización en farmacia clínica

Milena Prando Tupan Basségio ${ }^{1}$ Christian Boller², Gracinda Maria D’Almeida e

Oliveira $^{3}$, Maria Rosa Machado Prado ${ }^{4}$

${ }^{1}$ Mestre no Ensino nas Ciências da Saúde pela Faculdades Pequeno Príncipe- FPP Curitiba - Paraná

2 Doutor em Biotecnologia Aplicada à Saúde da Criança e do Adolescente. Docente do Programa de Mestrado em Ensino nas Ciências da Saúde da Faculdades Pequeno Príncipe Curitiba - Paraná

${ }^{3}$ Doutora em Odontologia pela Pontifícia Universidade Católica do Paraná . Curitiba - Paraná

${ }^{4}$ Doutora em Engenharia de Bioprocessos e Biotecnologia . Docente do Programa de Mestrado em Ensino nas Ciências da Saúde da Faculdades Pequeno Príncipe . Curitiba - Paraná

\section{RESUMO}

A Farmácia Clínica é uma área de atuação farmacêutica que tem crescido na última década, em especial após a publicação das Diretrizes Curriculares no ano de 2002. O objetivo deste trabalho 
foi avaliar a percepção de estudantes de pós-graduação em Farmácia Clínica sobre a sua formação acadêmica e o desenvolvimento de competência para o trabalho com Farmácia Clínica. Os participantes deste estudo foram estudantes de pós-graduação regularmente matriculados em Instituições de Ensino Superior, no Estado do Paraná. Os dados coletados passaram por análise de conteúdo e divididos em duas categorias: Ensino em Farmácia e Gestão Clínica. O resultado aponta para a existência de lacunas no processo de ensino e aprendizagem no que diz respeito ao cuidado com o paciente e no desenvolvimento de competências profissionais, demanda que, se suprida, permite valorização da profissão farmacêutica no concorrido mercado de trabalho.

Palavras-chave: Currículo. Educação em Farmácia. Competência Clínica

\begin{abstract}
Clinical Pharmacy is an area of pharmaceutical practice that has grown in the last decade, especially after the publication of the Curriculum Guidelines in 2002. The objective of this work was to evaluate the perception of Clinical Pharmacy postgraduate students about their education, and the development of competence for work with Clinical Pharmacy. Participants in this study were graduate students regularly enrolled in higher education institutions, one located in the State of Paraná. Collected data underwent content analysis, and were divided into two categories: Pharmacy Teaching and Clinical Management. The result points to the existence of gaps in the teaching and learning process with regard to patient care and the development of professional skills, a demand that, if fulfilled, allows the valuation of the pharmaceutical profession in the crowded labor market.
\end{abstract}

Keywords: Curriculum. Education, Pharmacy . Clinical Competence.

\title{
RESUMEN
}

La Farmacia clínica es un área de práctica farmacéutica que ha crecido en la última década, especialmente después de la publicación de las Directrices Curriculares en 2002. El objetivo de este trabajo fue evaluar la percepción de los estudiantes de posgrado en Farmacia clínica sobre su educación y el desarrollo de competencias para trabajar con Farmacia Clínica. Los participantes en este estudio fueron estudiantes de posgrado inscriptos regularmente en instituciones de educación superior, una ubicada en Curitiba y otra en el suroeste del estado de Paraná. Los datos recopilados pasaron el análisis de contenido y se dividieron en dos categorías: enseñanza de farmacia y gestión clínica. El resultado apunta a la existencia de brechas en el proceso de enseñanza y aprendizaje con respecto a la atención al paciente y al desarrollo de habilidades profesionales, lo que, si se proporciona, permite la valoración de la profesión farmacéutica en el concurrido mercado laboral.

Palabras clave: Curriculum. Educación en Farmacia. Competencia Clínica. 


\section{INTRODUÇÃO}

A Universidade é uma instituição inserida em um contexto sociopolítico específico, localizada espacial e temporalmente. Portanto, além do ato de ensinar, existe uma concepção ideológica que visa atender diferentes demandas sociais, seja na graduação ou pós-graduação $0^{1,2}$.

Optar por um curso superior envolve uma série de fatores como, o modelo de gestão, custo (privado), renome da instituição, possibilidade de intercâmbios estudantis, opções de estudo da grade curricular, entre outros. Neste aspecto, o acesso à Universidade aumentou, em especial no final do século XX, com a superlotação das Instituições públicas e o aumento da oferta de cursos em Instituições privadas ${ }^{2,3}$.

Além dos pontos destacados anteriormente, a escolha de uma Instituição de Ensino Superior (IES), por um futuro estudante, perpassa os objetivos pedagógicos do curso (contemplados no Projeto Político Pedagógico) e as oportunidades de complementação da formação após a conclusão do Curso Superior. Dessa forma, a escolha de um curso universitário é guiada, em geral, pela expectativa de uma atuação profissional promissora social em detrimento da aptidão pessoal ${ }^{2}$.

O Curso de Farmácia é exemplo vivo das mudanças sociais, culturais, políticas e econômicas observadas nos últimos 100 anos na sociedade Brasileira. Um diálogo comum dos educadores em farmácia aponta que a educação farmacêutica do século XXI mostrou algumas mudanças como: 1) aumento do número de IES que ofertam o curso, 2) aumento da diversidade das matrizes curriculares, 3) mudanças globais no escopo da prática farmacêutica com maior atenção às necessidades locais, 4) aumento da disponibilidade de diferentes tecnologias incluindo informação, comunicação e educação, 5) melhor compreensão do estilo de aprendizagem do estudante, o que leva em consideração também a motivação e efetividade do ensino, 6) crescimento mundial da preocupação com a qualidade da educação farmacêutica ${ }^{4}$.
Estas características foram observadas inicialmente, no Brasil, com a implantação da Diretriz Curricular Nacional de 2002 e da consagração do Sistema Único de Saúde (SUS), oportunizando ao profissional farmacêutico uma carreira ligada à prática clínica. Neste contexto ${ }^{5}$, destaca-se que estas modificações no exercício da profissão farmacêutica exigem novos conhecimentos, habilidades e atitudes para atender as preocupações, necessidades e expectativas da sociedade. Como destacado, tanto o farmacêutico quanto a Universidade necessitaram se adaptar para atender os serviços de saúde que a sociedade atual carece.

Este novo perfil de profissional exigido pela sociedade do século XXI leva o farmacêutico a preocupar-se mais com os determinantes e condicionantes de saúde, e a entender a saúde como completo estado de bem-estar físico, mental e social $^{6}$, e assim superar os conhecimentos técnicos das análises clínicas ou da produção magistral e industrial de medicamentos, que têm como premissa a saúde como ausência de doença ou enfermidade ${ }^{7}$. Esta concepção leva o farmacêutico a trabalhar na perspectiva do uso racional de medicamentos e em conjunto com a equipe de saúde ${ }^{8}$.

Desta forma, o presente trabalho tem como objetivos avaliar a percepção de estudantes de pós-graduação em Farmácia Clínica sobre a sua formação acadêmica nesta área e o desenvolvimento de competência para o trabalho com Farmácia Clínica.

Este estudo visa contribuir para a formação acadêmica dos futuros farmacêuticos, uma vez que o mercado de trabalho tem exigido profissionais com competência para atuar, intervir e prevenir problemas relacionados a medicamentos. Este tema é amplamente abordado dentro do contexto da farmácia clínica e tem sido cada vez mais exigido na formação acadêmica. 


\section{O olhar sobre a formação acadêmica}

O ensino tradicional de Farmácia se concentrou, por muito tempo, na retenção de informações e repetição de conteúdos básicos, sem contemplar a resolução de problemas, necessária para reforçar o pensamento crítico. Isso trouxe, como consequência, estudantes pouco preparados para os problemas da vida real ${ }^{9}$.

Várias propostas pedagógicas inovadoras surgiram como alternativas ao ensino tradicional, como a metodologia da problematização, a aprendizagem baseada em problemas (PBL), exame clínico objetivo estruturado, jogos, simulação, entre outros. Esta forma de ensino tem sido adotada como forma de reduzir a distância entre a academia e prática profissional ${ }^{10-12}$.

Esta nova forma de compreender a realidade da formação farmacêutica iniciou-se nos Estados Unidos na metade do séc. XIX com a adoção de metodologias ativas de ensino e aprendizagem. No Brasil, este modelo foi organizado e tem sido implantado gradativamente, principalmente após a publicação das Diretrizes Curriculares do Curso (DCN) de Farmácia em 2002 e reforçadas na DCN de 2017. Estes documentos legais apontam para a necessidade de uma formação contínua, estendendo o período de formação por toda a vida profissional ${ }^{13}$.

Um conceito importante apresentado pelas Diretrizes é o cuidado em saúde, que é o conjunto de ações e de serviços ofertados ao indivíduo, à família e à comunidade, que considera a autonomia do ser humano, a sua singularidade e o contexto real em que vive, sendo realizado por meio de atividades de promoção, proteção e recuperação da saúde, além da prevenção de doenças, e que possibilite às pessoas viverem melhor ${ }^{14}$.

As atribuições clínicas do farmacêutico já são realidade em grande parte dos países de Primeiro Mundo. No Brasil, elas passaram a reverberar, com mais intensidade, nos últimos dez anos, e estão se organizando como um movimento que poderá estar na vanguarda das mudanças aguardadas para a saúde e tem merecido a atenção das autoridades sanitárias ${ }^{15}$.

\section{Matriz de competência para formação do farmacêutico}

A área de Farmácia Clínica é norteadora para o farmacêutico no cuidado direto ao paciente, conduzindo-o à prática do uso racional de medicamentos, de forma a otimizar a farmacoterapia, promover saúde e bem-estar, e prevenir doenças e outras condições. Ela, como prática profissional, se estrutura a partir dos componentes: modelo de prática, filosofia ou princípios da prática; o processo de cuidado e a gestão da prática ${ }^{16-17}$.

Em relação ao processo de ensino, aprendizagem e avaliação de competências clínicas, o marco inicial da proposta de padrões mínimos baseia-se na matriz de competências da DCNs de 2017, que é um instrumento norteador para a formação clínica de farmacêuticos. Destaca-se que a definição de uma matriz de competências constitui apenas a primeira etapa de um processo educacional. Vale salientar que o processo de ensino-aprendizagem e sua avaliação, necessitam de aplicação contextualizada com a concepção teórica de competência e de estruturação consciente $\mathrm{e}^{18-19}$.

A estruturação do processo de ensino-aprendizagem deve resultar de planejamento que contemple a escolha do conteúdo, dos procedimentos, das atividades, dos recursos, das estratégias, dos cenários de prática ou lugares de aprendizagem, de sistemas de avaliação com enfoque formativo e somativo, e de feedback aos estudantes, entre outros. Apesar de implícito no processo de aprendizagem, esta definição deve ser previamente, no início da disciplina, e sua intencionalidade deve ser reconhecida pelo educador ${ }^{20}$.

Um processo de aprendizagem com modelagem 
pensada e definida de forma a contemplar estes elementos oportuniza a formação de farmacêuticos capazes de identificar e acolher demandas, determinar necessidades ou problemas de saúde dos pacientes, da família e da comunidade, delinear e implantar planos de cuidado e avaliar os resultados de sua aplicação.

\section{MÉTODo}

A presente pesquisa trata-se de um estudo exploratório e descritivo e de cunho qualitativo, e foi desenvolvido em duas Instituições de Ensino Superior, sendo uma na cidade de Curitiba e outra na região Oeste do Paraná.

A pesquisa foi constituída por 32 estudantes de Cursos e Especialização em Farmácia Clínica. A coleta de dados foi realizada com a aplicação de um questionário com perguntas abertas que versavam sobre: 1) dados demográficos, 2) distribuição dos conteúdos de Farmácia Clínica ao longo do curso de graduação, 3) carga horária (teórica e prática), 4) importância da atenção farmacêutica e a comunicação com os pacientes, 5) comunicação entre profissionais de saúde, 6) a segurança em promover o uso racional dos medicamentos, 7) contribuição do curso de graduação para uma intervenção farmacêutica efetiva e 8) confiança na tomada de decisões clínicas.

Os participantes foram previamente contatados e foi-lhes solicitado a assinatura de um Termo de Consentimento Livre e Esclarecido. Para garantir a confidencialidade das respostas e o anonimato dos participantes, os questionários receberam os seguintes códigos: IES1 para os participantes da Instituição de Ensino Superior de Curitiba e IES2 para os participantes da Instituição de Ensino Superior do Oeste do Paraná.

Para a análise das informações utilizou-se a técnica de análise de conteúdo temática proposta por Minayo $^{21}$, que busca a interpretação cifrada do material de caráter qualitativo. Esta técnica possui três etapas: A primeira é a Pré-Análise - onde os objetivos e hipóteses iniciais da pesquisa são retomados, e as respostas são organizadas de forma que possa permitir uma compreensão prévia do material teórico. A segunda etapa é a Exploração do Material: onde é realizada a operação classificatória com o intuito de alcançar o núcleo de compreensão do texto e estabelecer as categorias. A terceira etapa constitui o Tratamento dos Resultados Obtidos e interpretação, com interpretação das repostas dos participantes e inter-relacionando as mesmas com o quadro teórico.

O estudo atendeu os preceitos éticos da resolução no 466/12 da Comissão de Ética em Pesquisa em Seres Humanos e aprovados pelo parecer número 2.086.740.

\section{RESULTADOS}

Os questionários foram respondidos por 28 participantes do gênero feminino e 4 do gênero masculino. As idades dos farmacêuticos variaram entre 21 e 43 anos com média de 25 anos. Quando perguntados sobre sua graduação, vinte e quatro participantes responderam que cursaram farmácia em IES pública e oito em IES privada, todas do Estado do Paraná.

As respostas dos questionários foram transcritas e categorizadas em: Ensino em Farmácia e Gestão Clínica. A primeira categoria, ENSINO EM FARMÁCIA, apresentou três subcategorias e unidades de contexto de acordo com o quadro 1.

$\mathrm{Na}$ subcategoria 'Necessidade de aperfeiçoamento' foi abordado o real interesse dos egressos na busca por uma especialização. Neste contexto, observouse que a especialização proporciona possibilidade de ascensão profissional e aperfeiçoamento nos conhecimentos.Ainda nesta subcategoria foi pesquisada a importância de cursar disciplinas que capacitem o profissional para atuar na atividade 
clínica. Os relatos revelaram a preferência por conteúdos que forneçam base para a atuação na atividade clínica, com destaque para aqueles que aproximem o profissional da realidade do mercado.

Quadro 1 - Categoria ensino em farmácia clínica.

\begin{tabular}{|c|c|c|c|}
\hline \multirow{2}{*}{ Categoria } & \multicolumn{2}{|c|}{ Ensino em Farmácia } \\
\hline Subcategoria & Necessidade de aperfeiçoamento & Formação profissional & Formação Graduação \\
\hline $\begin{array}{c}\text { Unidades de } \\
\text { Contexto }\end{array}$ & $\begin{array}{c}\text { Expectativa de cursar farmácia } \\
\text { clínica }\end{array}$ & $\begin{array}{c}\text { Habilidade em gerenciar } \\
\text { conflitos } \\
\text { disciplina }\end{array}$ & $\begin{array}{c}\text { Carga horária teórico/ } \\
\text { prática }\end{array}$ \\
\hline
\end{tabular}

Fonte: Os autores.

Em relação à unidade de contexto "Expectativa de cursar farmácia clínica”, os participantes relatam um anseio positivo pelo Curso de Farmácia Clínica por haver possibilidade de complementar a formação adquirida no decorrer da graduação e o despertar profissional para a nova área de atuação ascendente no mercado de trabalho, como transcrito, literalmente, a partir dos relatos:

- A necessidade de ampliar os conhecimentos e oportunidades com o surgimento da prescrição farmacêutica (IES1-11).

- Buscar mais conhecimento e aperfeiçoamento, para fazer um diferencial na minha profissão farmacêutica, para aperfeiçoar os cuidados farmacêuticos e bem atender o paciente (IES1-12).

- Pela necessidade de atualização e demanda solicitada na instituição em que eu trabalho. Pelo desejo de obter mais um título na carreira e pela importância do serviço prestado pelo farmacêutico (IES2-06).

Já na Unidade de Contexto "Experiência ao cursar a disciplina” os participantes relataram as atividades desenvolvidas e os conteúdos ministrados, despertando o interesse pela área de atuação e proporcionando segurança, aptidão e capacitação profissional para os processos clínicos, como demonstrados a seguir:

- Contribui para um melhor conhecimento do profissional, aperfeiçoamento, segurança nas atividades atribuídas (IES1-04).

- Atuação clínica, com segurança e efetividade, buscando sempre o melhor ao paciente (IES2-02).

- Possibilitar uma melhor fundamentação teórica para aplicar ao campo de trabalho (IES2-07).

A subcategoria “formação profissional' envolve as descrições sobre a gestão da prática e a deficiência de estágios, gestão de pessoas que envolvem um relacionamento seguro para exercer uma comunicação objetiva, gestão da interdisciplinaridade para compreender e entender qual o seu papel dentro da equipe de saúde. Quando o assunto é voltado para o trabalho em equipe e gerenciar conflitos, percebe-se na opinião do egresso a necessidade do incentivo por parte da instituição para uma melhor comunicação com as outras áreas de saúde, além de treinamentos e padronização dos processos no ambiente de trabalho. 
A partir da Unidade de Contexto "habilidades em gerenciar conflitos" compreendem-se pelos relatos que, para muitos participantes, houve deficiência de integração das atribuições clínicas do profissional com a equipe de saúde durante a formação, e também relataram serem insatisfatórios o contato e a comunicação com os outros profissionais impossibilitando sua real participação na equipe multiprofissional:

- Além dos conteúdos abordados em aula, teríamos que ter estágio de qualidade e programas, cursos ofertados pela instituição. (IES1-05)

- Deveria ser incorporado já na formação incentivando o aumento da interação entre profissionais. Exercer uma boa comunicação com a equipe, realizar treinamentos. (IES1-08)

- Acredito que a parte de gestão e interdisciplinaridade é de suma importância p/ que o farmacêutico entenda qual é o papel dentro da equipe. (IES2-06)

A subcategoria "formação graduação" compreende o estudo da farmácia clínica durante o curso de farmácia e se os conteúdos e a carga horária foram consideráveis. Quanto à Unidade de Contexto "Carga horária teórica e prática", os participantes descreveram que a farmácia clínica foi abordada de forma muito superficial durante o curso, não como uma disciplina, e sim como um conteúdo dentro de outras disciplinas, como, por exemplo, a atenção farmacêutica. Em consideração à carga horária, os participantes declararam que ela é insatisfatória tanto em teoria como em prática, o que levou à busca pela especialização.

- Na época da minha graduação não teve matérias especificas para farmácia clínica, a matéria mais próxima de farmácia clínica que dava uma introdução superficial a esta área era a atenção farmacêutica. (IES1-02)

- A farmácia clínica na faculdade foi abordada apenas na matéria de atenção farmacêutica I e II. Os conteúdos foram excelentes, porém a carga horária deveria ser ampliada. (IES2-12)

- Durante a graduação teve disciplinas que abordaram a atenção farmacêutica, porém ainda era um assunto mais abordado para hospital, gostaria de ter me aprofundado mais, por essa razão busquei especialização. (IES2-15)

A segunda categoria nomeada como "gestão clínica" possui como subcategorias atuação clínica, gerenciar informações e intervenção farmacêutica, que correspondem às unidades de contexto: comunicação de profissionais de saúde e pacientes, promover o uso racional de medicamentos e capacidade de tomada de decisão, conforme quadro 2.

Quadro 2 - Categoria Gestão Clínica.

\begin{tabular}{|c|c|c|c|}
\hline Categoria & \multicolumn{2}{|c|}{ Gestão Clínica } \\
\hline Subcategoria & Atuação Clínica & Gerenciar Informações & $\begin{array}{c}\text { Intervenção } \\
\text { Farmacêutica }\end{array}$ \\
\hline Unidades de Contexto & $\begin{array}{c}\text { Habilidade de } \\
\text { Comunicação }\end{array}$ & $\begin{array}{c}\text { Promover uso racional de } \\
\text { medicamentos }\end{array}$ & $\begin{array}{c}\text { Capacidade de tomada } \\
\text { de decisões }\end{array}$ \\
\hline
\end{tabular}

Fonte: Os autores. 
A subcategoria "atuação clínica" denota a relação da prática entre os profissionais de saúde e a capacidade de comunicação efetiva com o paciente. Também se destaca o processo de humanização, que proporciona um melhor atendimento, mais completo e personalizado, e na unidade de contexto "Habilidade de comunicação", como apresentado nos relatos, os participantes apontam a necessidade de uma comunicação clara e compartilhada entre os profissionais de saúde, para que se obtenha uma prestação de serviços orientada aos pacientes, promovendo um atendimento eficiente e completo.

- Sim, com uma comunicação clara e objetiva é possivel um bom desenvolvimento farmacoterapêutico. (IES1-08)

- Sim, tenho um bom relacionamento com os outros profissionais e com os pacientes, sendo objetiva no modo de passar as orientações. (IES1-11)

- Sim, um trabalho em farmácia clínica requer sempre o apoio do corpo clínico das instituições. (IES2-02)

$\mathrm{Na}$ subcategoria "Gerenciar Informações", os participantes relataram sobre a promoção do uso racional de medicamentos e o eficiente manejo do mesmo. Na unidade de contexto "promover o uso racional de medicamentos", os relatos, em sua maioria, retratam as atividades clínicas desenvolvidas com segurança e eficiência, como também avaliam cada situação e com materiais de apoio conseguem promover com qualidade o atendimento aos pacientes. Relatam também a importância da experiência, integração com profissionais de saúde e a capacitação continuada para um tratamento farmacoterapêutico mais efetivo.

- Sinto um pouco de insegurança, pois há a necessidade de abordar a farmácia clínica de um modo mais efetivo e amplo durante a graduação. (IES1-11)

- Sim. Leio a receita, analiso os medicamentos, a dosagem posológica, estando correto, oriento paciente a tomar e adequar ao melhor horário para que o tratamento seja bem sucedido. (IES1-14)
- Sim, porém sempre teremos que nos atualizar nos assuntos e estudos para que haja uma maior segurança para os pacientes. (IES2-04)

$\mathrm{Na}$ subcategoria 'Intervenção Farmacêutica', é abordado se, após a graduação, os entrevistados são capazes de interpretar e utilizar dados laboratoriais, permitindo uma intervenção farmacêutica por parte do profissional. Na unidade de contexto "Capacidade de tomada de decisão", os participantes destacam que em algumas situações eles possuem segurança para o atendimento farmacoterapêutico.

- Depende da situação, alguns assuntos são vistos superficialmente na graduação.(IES1-08)

- Sim, em algumas situações, mas ainda há muito que aprender, é necessário estar sempre buscando novos conhecimentos. (IES1-10)

- Sim, consigo em determinadas situações, mas sinto a necessidade de ampliar os conhecimentos. (IES1-11)

- Sim, mas sempre com apoio de algum material de apoio ou profissional, pois saímos da faculdade apenas com a teoria. (IES2-02)

\section{DISCUSSÃo}

Por meio dos resultados obtidos, foi possível apreender a percepção dos participantes em relação à disciplina de Farmácia Clínica durante a sua formação acadêmica, que atende as competências necessárias referentes às suas atribuições clínicas, mas não é capaz de dar segurança ao estudante no desenvolvimento desta atividade. Observouse, também, que os participantes têm pouco contato com conteúdos de Farmácia Clínica. Assim, demonstram interesse em se capacitarem, aprimorarem o conhecimento e as habilidades sobre as atribuições clínicas para o mercado de trabalho e, consequentemente, o despertar do interesse para a especialização ${ }^{22}$. 
Segundo a Organização Mundial da Saúde (OMS), a forma mais efetiva de prevenir o uso incorreto de medicamentos na atenção primária em países em desenvolvimento é a combinação de educação e supervisão dos profissionais de saúde, educação do consumidor e garantia de adequado acesso a medicamentos ${ }^{14,23}$. Quando analisadas as respostas dos participantes, elas apontam para as necessidades de uma base mais sólida entre teoria e prática na educação, contribuindo, assim, para uma melhoria nos processos de atendimento à população no que se refere ao uso dos medicamentos.

Os relatos apontam dúvidas sobre as tomadas de decisão em relação às intervenções farmacêuticas. Estas estão relacionadas à falta de segurança do profissional na aplicação das habilidades adquiridas durante a graduação e a aceitação da responsabilidade clínica do farmacêutico na garantia da farmacoterapêutica.

Vieira ${ }^{14}$ e Pádula ${ }^{24}$ descrevem que o farmacêutico passou, nos últimos 50 anos, por um modelo de ensino tecnicista, o que levou à desumanização da profissão. Os dois autores reiteram que o acolhimento ao paciente e a integração com a equipe de saúde são formas de aumentar a atuação do farmacêutico no contexto da saúde, incluindo-se aí o trabalho desenvolvido na Atenção Farmacêutica e Farmácia Clínica.

Por acolhimento do paciente entende-se a garantia da acessibilidade ao medicamento, atendimento eficaz do paciente com enfoque na farmacoterapia, ênfase no atendimento de pacientes em situação de risco medicamentoso, e oferecer atendimento comunitário como forma de ampliar a rede de atenção à saúde ${ }^{25}$.

No que tange ao trabalho em equipe, destaca-se a atuação profissional coletiva com reciprocidade na interação dos agentes. Quando se transpõe o trabalho de equipe dentro do ambiente de assistência à saúde, é importante haver flexibilidade na divisão do trabalho, respeitando dessa forma a autonomia técnica de cada profissional. Essas são as principais características do que é chamado de equipe integrada ${ }^{26}$.

Ainda em relação ao trabalho em equipe, a Organização Panamericana de Saúde (OPAS) entende isto como ato planejado, documentado e realizado junto ao usuário e profissionais de saúde, que visam prevenir ou resolver problemas que interferem ou podem interferir na farmacoterapia, sendo parte integrante do processo de acompanhamento, seguimento farmacoterapêutico ${ }^{27}$.

\section{CONCLUSÕES}

Este trabalho mostra que a profissão farmacêutica, em especial sua atuação clínica, tem visibilidade crescente nos últimos anos. Apesar disso, os participantes do estudo sentem que a IES poderia contribuir mais em sua formação, favorecendo atividades que aumentem a confiança no trabalho de farmácia clínica.

Farmacêuticos são e serão importantes sempre que exista a necessidade de uso do medicamento: na drogaria, na unidade de saúde, no hospital, nas Equipes de Saúde da Família. Mas para isso acontecer, a formação dos novos profissionais deve ser repensada e adequada às necessidades da sociedade. Uma educação estagnada no tempo não consegue preparar o profissional para os desafios do futuro, desta forma ela deve estar atrelada às mudanças sociais, dentro da qual se dê um valor significativo ao reconhecimento da realidade local. Isto permitirá ao farmacêutico transformar a sua realidade profissional e promover os princípios de uma saúde voltada ao atendimento das necessidades da sociedade. 


\section{REFERÊNCIAS}

Oliven AC. A marca da origem: comparando colleges americanos e faculdades brasileiras. Cad. Pesqui. 2005; 35(125): 111-135.

Severino, AJ. O ensino superior no Brasil: novas configurações e velhos desafios. Educar. 2008; 31: 73-89.

Braghini KMZ. A história dos estudantes "excedentes" nos anos 1960: a superlotação das universidades e um "torvelinho de situações improvisadas". Educar em Revista. 2014; 51: 123-144.

Fathelrahman AI, Ibrahim MIM, Alrasheedy AA, Wertheimer AI. Pharmacy education in the twenty first century and beyond. London: Elsevier; 2018.

Zubioli A. Farmácia clínica: um novo rumo para a profissão. O farmacêutico em revista. 2017; 116: 14-23.

Carrapato P, Correia P, Garcia B. Determinante da saúde no Brasil: a procura da equidade na saúde. Saúde e Sociedade. 2017; 3: 676-689.

Del Corral FSD, Souza MLA, Negrão OL. Do boticário ao farmacêutico: o ensino de farmácia na Bahia de 1815 a 1949. Salvador: EDUFBA; 2009.

Zubioli A. A farmácia clínica na farmácia comunitária. Brasília: Ethosfarma; 2001.

Blouin RA, Joyner PU, Pollack GM. Preparing for a renaissance in pharmacy education: the need, opportunity, and capacity for change. Am J Pharm Educ. 2008; 72(2): 1-3.

Berbel NAN. A problematização e a aprendizagem baseada em problemas: diferentes termos ou diferentes caminhos? Interface. 1998; 2(2):139-54.

Waterfield J. Is pharmacy a knowledge-based profession? Am j pharm educ. 2010; 74(3): 1-6.

Salitrini FD, O'Connell MB, Garwood CL, Lehr VT, Abdallah K. An objective structured clinical examination to assess problem-based learning. Am j pharm educ. 2012; 76(3): 1-10.

Vieira FS. Possibilidades de contribuição do farmacêutico para a promoção à saúde. Ciênc saúde coletiva. 2007; 12(1): 213-220.
Brasil. Resolução CNE/CES 2, de 19 de Outubro de 2017. Institui Diretrizes Curriculares Nacionais do Curso de Graduação em Farmácia e da outras providências. Diário Oficial da União, Brasília, DF, 20 de outubro de 2017.

João WJ. Prescrição farmacêutica e atribuições clínicas: para a história da profissão. Pharmacia brasileira. 2014; 88: 4-5.

Cipolle R, Strand LM, Morley PC. El ejercício de la atención farmaceutica. Madrid: McGraw Hill / Interamericana; 2000.

Fernandes ZC, Bermand MD, Oliveira Filho NCF, Camargo EES, Cecy C. Os desafios da educação farmacêutica no Brasil. Brasília: Conselho Federal de Farmácia; 2008.

Galato D, Alano, GM, Trauthman SC, Vieira AC. A dispensação de medicamentos: uma reflexão sobre o processo para prevenção, identificação e resolução de problemas relacionados à farmacoterapia. Rev bras cienc farm. 2008; 44(3): 465-475.

Gontijo ED, Alvim C, Megale L, Melo JRC, Lima MECC. Matriz de competências essenciais para a formação e avaliação de desempenho de estudantes de medicina. Rev bras educ med. 2013; 37(4): 526-539.

González AD, Almeida MJ. Integralidade da saúde: norteando mudanças na graduação dos novos profissionais. Cienc saúde coletiva. 2010; 15(3): 757-762.

Minayo MCS. O desafio do conhecimento: pesquisa qualitativa em saúde. 14. ed. São Paulo: Hucitec; 2014.

Salen GB, Rezk NL, Laika L., Ali A., El-Metwally A. Pharmacist, the pharmaceutical industry and pharmacy education in Saudi Arabia: A questionnaire-based study. Saudi Pharmaceutical Journal. 2015; 23; 573-580.

Tempsk P, Borba M. O SUS como escola. Rev bras educ med. 2009; 33(3): 319-320.

Pádula M, Pinto AV, Matos GC, Siqueira DT, Vieira RC. Atenção farmacêutica e atenção flutuante: formações de compromisso entre farmácia e psicanálise. Ciênc Saúde Coletiva. 2014; 19(2): 609-618.

Araújo ALA, Pereira LRL, Ueta, JM, Freitas O. Perfil da assistência farmacêutica na atenção primária do SUS. Ciênc Saúde Coletiva. 2008; 13: 611-617. 
Peduzzi M. Equipe multiprofissional de saúde: conceito e tipologia. Rev. Saúde Pública. 2001; 35(1): 103-109.

OPAS (Organização Pan-Americana de Saúde). Atenção farmacêutica no Brasil: trilhando caminhos. Relatório 2001-2002. Brasília: Distrito Federal; 2002. 